\title{
A Role for Iron in Germination of Conidia of Colletotrichum musae
}

\author{
By DAVID B. HARPER,* T. R. SWINBURNE, SOLVEIG K. MOORE, \\ AVERIL E. BROWN AND HADDEN GRAHAM \\ Faculty of Agriculture, The Queen's University of Belfast, Newforge Lane, Belfast BT9 5PX, \\ Northern Ireland, and Department of Agriculture, Northern Ireland
}

(Received 7 January 1980; revised 22 April 1980)

\begin{abstract}
The time course of germination of conidia of Colletotrichum musae in the presence of different germination stimulants is consistent with the postulate that the stimulation of germination by anthranilic acid can be attributed to its previously established conversion to 2,3-dihydroxybenzoic acid in conidia. The activity of a number of synthetic chelating agents, such as EDTA and 1,10-phenanthroline, in promoting germination of the same order as 2,3-dihydroxybenzoic acid at about the same molarity provides support for the hypothesis that the chelating properties of the latter compound are important in its biological action.

Experiments with conidia produced on media of different iron concentrations indicate that in the absence of a germination stimulant there is a high inverse correlation between the percentage germination of conidia and conidial iron content. However, in the presence of anthranilic acid there is no significant correlation between these parameters, percentage germination remaining uniformly high regardless of the conidial iron concentration. It is concluded that conidial iron is involved in the inhibition of germination and that this inhibition can be relieved non-specifically by a variety of chelating agents.
\end{abstract}

\section{INTRODUCTION}

Previously, Swinburne (1976) established that anthranilic acid found in leachates of banana fruit stimulates germination of conidia of Colletotrichum musae, a pathogen responsible for anthracnose lesions on bananas. Subsequent studies by Harper \& Swinburne (1979) demonstrated that anthranilic acid was degraded by both mycelium and conidia of C. musae mainly to 2,3-dihydroxybenzoic acid (DHBA). As DHBA was found to be a more effective stimulant of germination than anthranilic acid, they suggested that the latter compound was not active per se in promoting germination but only by virtue of its ready conversion to DHBA. Further evidence in support of this hypothesis is presented.

The chelating properties of DHBA and its conjugates play a vital role in their welldocumented function in iron transport in bacteria (Lankford, 1973; Neilands, 1973). Indeed, siderophores produced by saprophytic bacteria can also act as stimulants of germination in C. musae (McCracken \& Swinburne, 1979). In view of these observations, Harper \& Swinburne (1979) proposed that the action of DHBA in stimulating germination in C. musae might similarly depend on its chelating abilities, and that iron was intimately involved in the process. DHBA may function by influencing either uptake, release or intracellular transport of iron prior to germination. However, the finding that only siderophores in the desferri form were active in promoting germination (McCracken $\&$ Swinburne, 1979) suggested that iron uptake was not involved. 
In the present paper we describe experiments to verify the proposition that the process of chelation is important in the stimulation of germination and to confirm the participation of iron.

\section{METHODS}

Cultural conditions. Colletotrichum musae was isolated from anthracnose lesions on imported bananas and maintained on modified Cook's no. 2 agar medium as described previously (Swinburne, 1976). Washed suspensions of conidia harvested from 5 to $7 \mathrm{~d}$-old colonies grown at $20^{\circ} \mathrm{C}$ were used in germination assays.

The concentration of iron in the agar used in the preparation of the above medium was relatively high (about 500 p.p.m.), and so for experiments requiring conidia produced from mycelium grown on media of low or precisely defined iron concentration, conidia were harvested from mycelium grown in liquid shake cultures on iron-depleted media prepared as follows. Asparagine $(15 \mathrm{~g})$ and glucose $(20 \mathrm{~g})$ were dissolved in distilled water (1 1 ) and the solution was passed three times down a column $(22.5 \times 5.0 \mathrm{~cm})$ of Chelex 100 (Bio-Rad) prepared in the sodium form at $\mathrm{pH} 6.3$ by rinsing with four bed volumes of $0.5 \mathrm{M}$-sodium acetate buffer. The following analytical grade reagents were added to the iron-depleted eluate from the column: $\mathrm{MgSO}_{4} .7 \mathrm{H}_{2} \mathrm{O}, 0.5 \mathrm{~g} ; \mathrm{K}_{2} \mathrm{HPO}_{4}, 0.5 \mathrm{~g} ; \mathrm{CaCl}_{2} .6 \mathrm{H}_{2} \mathrm{O}, 0.2 \mathrm{~g}$. The $\mathrm{pH}$ of the solution was adjusted to 6.0 and the medium was supplemented with a trace element solution $(4 \mathrm{ml})$ containing the following analytical grade reagents in distilled water (1 1): $\mathrm{ZnSO}_{4} .7 \mathrm{H}_{2} \mathrm{O}, 0.22 \mathrm{~g}$; $\mathrm{CuSO}_{4} .5 \mathrm{H}_{2} \mathrm{O}, 0.04 \mathrm{~g} ; \mathrm{MnSO}_{4} .4 \mathrm{H}_{2} \mathrm{O}, 0.02 \mathrm{~g}$; $\mathrm{Na}_{2} \mathrm{MoO}_{4} .2 \mathrm{H}_{2} \mathrm{O}, 0.02 \mathrm{~g}$. The iron content of this medium, as determined by atomic absorption spectrophotometry, was usually in the range 9 to $12 \mathrm{ng} \mathrm{ml}^{-1}$. Media containing a range of iron concentrations up to $500 \mathrm{ng} \mathrm{ml}^{-1}$ were prepared by adding $\mathrm{FeSO}_{4} .7 \mathrm{H}_{2} \mathrm{O}$ solution $\left(0 \cdot 26 \mathrm{~g}^{-1}\right)$ to this iron-depleted medium. All glassware used in the preparation of low iron media and subsequent culture and manipulation of mycelia and conidia was scrupulously cleaned, soaked overnight in $0.5 \mathrm{M}-\mathrm{HCl}$ and washed with demineralized water prior to use, to minimize the risk of iron contamination. Portions $(100 \mathrm{ml})$ of the medium were dispensed into conical flasks $(250 \mathrm{ml})$, sterilized and, after inoculation with conidial suspension $(0.5 \mathrm{ml})$, incubated at $21^{\circ} \mathrm{C}$ in an orbital shaker with an eccentricity of $2.5 \mathrm{~cm}$ at $120 \mathrm{rev}$. $\mathrm{min}^{-1}$. After $4 \mathrm{~d}$, conidia were obtained by filtering the contents of each flask through glass wool to remove mycelium and centrifuging the remaining conidial suspension. The conidia were centrifuged and resuspended in water twice before use. The inoculum for such liquid cultures was a conidial suspension harvested from mycelium grown for at least two generations in liquid culture medium containing $40 \mathrm{ng}$ iron $\mathrm{ml}^{-1}$. This precaution precluded the possibility of significant concentrations of iron being transferred in the inoculum to the fresh culture medium.

Germination assay. Washed conidia obtained from cultures on solid medium were used to assay the effectiveness of synthetic and natural iron-chelating compounds as germination stimulants. In such assays, conidial suspensions $\left(6 \times 10^{5}\right.$ to $7 \times 10^{5}$ conidia $\left.\mathrm{ml}^{-1}\right)$ were mixed with the appropriate test solution or water and duplicate drops $(10 \mu \mathrm{l})$ were placed on glass slides and incubated in a moist chamber at $22^{\circ} \mathrm{C}$ for $18 \mathrm{~h}$. Compounds were tested at a range of concentrations between $10^{-3}$ and $10^{-6} \mathrm{M}$, the $\mathrm{pH}$ of the solutions having been adjusted to 5.0 with either $\mathrm{HCl}$ or $\mathrm{NaOH}$. Counts for germination were made on at least 200 randomly selected conidia in each drop. Each experiment was repeated at least three times. The concentration at which each compound was most effective as a germination stimulant was determined and the percentage germination at that concentration was calculated.

Assay of conidial iron. The concentration of iron in conidia was determined both by atomic absorption spectrophotometry and by a method involving the use of ${ }^{59} \mathrm{Fe}$. Conidia produced under different iron regimens were separated from mycelium by filtration through glass wool and harvested by centrifugation at $2000 \mathrm{~g}$ for $5 \mathrm{~min}$. After being washed twice by resuspension in water and recentrifugation, the conidia were finally suspended in water $(100 \mathrm{ml})$ in a volumetric flask. Samples from this suspension were assayed for iron using a Perkin Elmer 306 atomic absorption spectrophotometer fitted with an HGA 72 heated graphite atomizer. Dry weights of samples of suspension were determined by drying at $80^{\circ} \mathrm{C}$ to constant weight and the iron concentration of conidia was expressed on a dry weight basis.

Conidia for assay by the radioisotopic technique were produced in liquid cultures as described above except for the replacement of $10 \mathrm{ng}$ iron $\mathrm{ml}^{-1}$ in the medium by ${ }^{59} \mathrm{FeCl}_{\mathrm{a}}$ [sp.act. $5 \mu \mathrm{Ci}_{\mu \mathrm{g}}^{-1}\left(185 \mathrm{kBq} \mathrm{g}^{-1}\right)$ ]. Samples $(250 \mu \mathrm{l})$ of washed conidial suspensions were added to NE250 scintillation fluid $(5 \mathrm{ml}$, Nuclear Enterprises, Edinburgh) and counted using an ICN Tracerlab Coru/matic 200 scintillation counter. From such counts and the known specific activity of iron in the culture medium prior to inoculation, the iron concentration of the suspension could be calculated since localization of iron within conidia did not lead to any apparent increase in quenching.

Atomic absorption spectrophotometry gave reproducible results in the determination of iron in the culture medium but was less consistent than the radioisotopic method when employed to assay iron in conidia, particularly at low iron concentrations. Sampling difficulties and extraneous contamination are probably responsible for such variability. Thus, conidial iron concentrations shown in Figs 4 and 5 are the means of four determinations using the more reliable labelling technique. 
Table 1. Comparison of some synthetic iron chelating compounds with DHBA and anthranilic acid as stimulants of germination of C. musae

Compound tested

8-Hydroxy-7-iodoquinoline-5-sulphonic acid Anthranilic acid

EDTA

2,3-Dihydroxybenzoic acid (DHBA)

1,10-Phenanthroline

Ammonium pyrollidine dithiocarbamate

Ethylenediamine dihydrochloride

8-Hydroxyquinoline

4,7-Diphenyl-1,10-phenanthroline

Control

$\begin{array}{cc}\begin{array}{c}\text { Optimum concn } \\ (\mathrm{M})\end{array} & \begin{array}{c}\text { Germination } \\ (\%)\end{array} \\ 5 \times 10^{-4} & 86 \pm 10^{*} \\ 10^{-3} & 79 \pm 11 \\ 5 \times 10^{-4} & 76 \pm 15 \\ 5 \times 10^{-4} & 71 \pm 12 \\ 10^{-4} & 70 \pm 10 \\ 5 \times 10^{-4} & 58 \pm 7 \\ 5 \times 10^{-4} & 58 \pm 22 \\ 5 \times 10^{-4} & 56 \pm 13 \\ 10^{-4} & 53 \pm 6 \\ - & 12 \pm 3\end{array}$

* Standard deviation of mean of three replicate experiments.

Comparison of time course of germination with different stimulants. The standard germination assay was modified to determine the time course of conidial germination with optimum concentrations of anthranilic acid, DHBA, EDTA and 8-hydroxy-7-iodoquinoline-5-sulphonic acid (HIQS). Washed conidial suspensions were mixed with the solution of the germination stimulant or water on a series of glass slides and incubated in a moist chamber at $22^{\circ} \mathrm{C}$. At hourly intervals over the next $16 \mathrm{~h}$, slides were removed from the chamber and counts for germination were made in the usual manner.

Exposure times necessary to achieve maximum germination. Experiments were performed to compare the lengths of time for which conidia had to be exposed to anthranilic acid, DHBA and other germination stimulants to obtain maximum enhancement of germination. Samples $(10 \mathrm{ml})$ of a suspension of washed conidia $\left(6 \times 10^{5}\right.$ to $\left.7 \times 10^{5} \mathrm{ml}^{-1}\right)$ were added to each of a series of conical flasks $(250 \mathrm{ml})$ containing $90 \mathrm{ml}$ solution of either anthranilic acid $\left(10^{-3} \mathrm{M}\right)$, DHBA $\left(5 \times 10^{-4} \mathrm{M}\right)$ or water. The flasks were incubated at $22^{\circ} \mathrm{C}$ on an orbital shaker at $60 \mathrm{rev} \cdot \mathrm{min}^{-1}$. At intervals of 30 to $60 \mathrm{~min}$ during the following $7 \mathrm{~h}$, samples $(10 \mu \mathrm{l})$ were taken from a flask of each treatment, placed on glass slides and assayed for germination following $18 \mathrm{~h}$ incubation in the normal manner. At each sampling time, one flask containing a suspension in anthranilic acid and one containing a suspension in DHBA were removed. The conidia from each flask were separated by filtration (Millipore, $0.45 \mu \mathrm{m}$ pore size), washed with water $(4 \times 25 \mathrm{ml})$ and resuspended in water $(10 \mathrm{ml})$. Drops $(10 \mu \mathrm{l})$ of this suspension were placed on glass slides, incubated in the normal manner and the percentage germination was assayed after a total of $18 \mathrm{~h}$ incubation. Sampling in this manner was continued for a period of 6 to $8 \mathrm{~h}$. Using this technique the germination of conidia immersed in germination stimulant for various relatively short periods of time could be compared with that of conidia immersed in germination stimulant for the complete $18 \mathrm{~h}$ of the normal assay.

\section{RESULTS}

\section{Chelating agents as stimulants of germination}

The concentrations at which each of the compounds tested were most stimulatory to germination of conidia of $C$. musae and the mean percentage germination achieved at that concentration are shown in Table 1 . All of the synthetic chelating agents tested were stimulatory; 8-hydroxy-7-iodoquinoline-5-sulphonic acid (HIQS), 1,10-phenanthroline and EDTA were outstanding in this respect. Almost all of the conidia germinating in HIQS or EDTA rapidly produced appressoria which were more rounded and regular in outline than those produced in water in which only 60 to $70 \%$ of the germinated conidia formed appressoria.

\section{Comparison of germination in anthranilic acid, DHBA, EDTA and HIQS}

The results of time course experiments which compared the rate of germination of conidia with optimum concentrations of anthranilic acid $\left(10^{-3} \mathrm{M}\right)$, DHBA $\left(5 \times 10^{-4} \mathrm{M}\right)$ and water are shown in Fig. 1. Germination in DHBA was first observed approximately $2 \mathrm{~h}$ earlier than in anthranilic acid. The subsequent rate of germination was slightly higher 


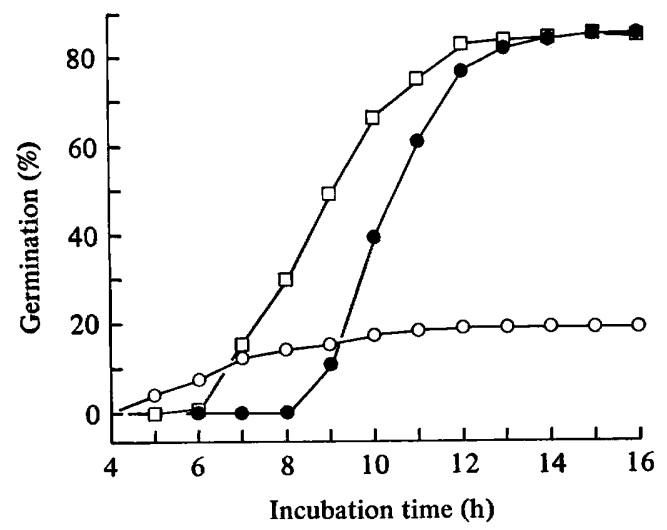

Fig. 1

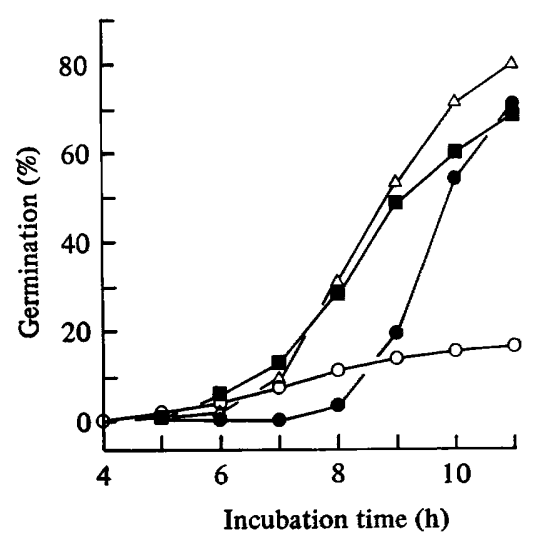

Fig. 2

Fig. 1. Germination (\%) of conidia of C. musae in $10^{-3} \mathrm{M}$-anthranilic acid (O), in $5 \times 10^{-4} \mathrm{M}^{-}$ DHBA $(\square)$ and in water $(O)$ as a function of time.

Fig. 2. Germination (\%) of conidia of $C$. musae in $5 \times 10^{-4} \mathrm{M}-8$-hydroxy-7-iodoquinoline-5-sulphonic acid $(\triangle)$, in $5 \times 10^{-4} \mathrm{M}$-EDTA $(\square)$, in $10^{-3} \mathrm{M}$-anthranilic acid $(O)$ and in water $(O)$ as a function of time.

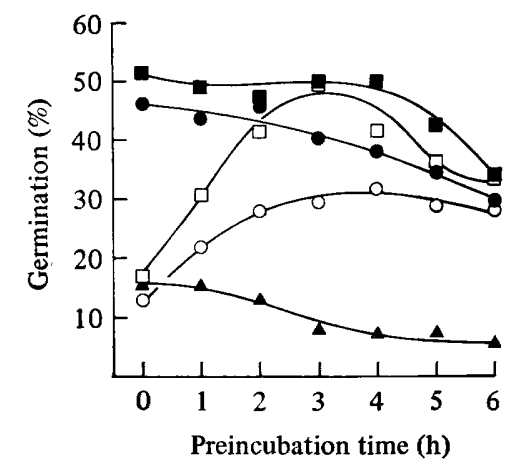

Fig. 3. Germination, after $18 \mathrm{~h}$ incubation, of conidia of $C$. musae following preincubation for various periods in germination stimulant. Preincubation in $5 \times 10^{-4} \mathrm{M}-\mathrm{DHBA}$ followed by incubation in $5 \times 10^{-4} \mathrm{M}$-DHBA ( $\square$ ), preincubation in $5 \times 10^{-4} \mathrm{M}-\mathrm{DHBA}$ followed by incubation in water $(\square)$, preincubation in $10^{-3} \mathrm{M}$-anthranilic acid followed by incubation in $10^{-3} \mathrm{M}$-anthranilic acid (O), preincubation in $10^{-3} \mathrm{M}$-anthranilic acid followed by incubation in water (O), preincubation and incubation in water $(\boldsymbol{A})$.

in anthranilic acid than in DHBA and maximum germination was achieved with both compounds after approximately the same period. Germination began in water earlier than with either of the compounds. In a similar experiment (Fig. 2), EDTA $\left(5 \times 10^{-4} \mathrm{M}\right)$, HIQS $\left(5 \times 10^{-4} \mathrm{M}\right)$ and anthranilic acid $\left(10^{-3} \mathrm{M}\right)$ were compared, and again germination was first observed with the chelating agents approximately $2 \mathrm{~h}$ earlier than with anthranilic acid, with maximum percentage germination achieved at about the same time with all compounds.

The results of experiments to compare the lengths of time for which conidia must be exposed to anthranilic acid or DHBA to obtain maximum enhancement of germination are shown in Fig. 3. After $2 \mathrm{~h}$ preincubation in DHBA, germination of conidia transferred to water was not significantly different $(P=0.01)$ from that of conidia in DHBA throughout. The corresponding preincubation period for anthranilic acid was $5 \mathrm{~h}$. The slight decline in apparent overall germination in DHBA, anthranilic acid and water during the course of the experiment can probably be attributed to a failure of the procedure employed to recover all the germinating conidia or, alternatively, to damage to a proportion of germinating conidia. 


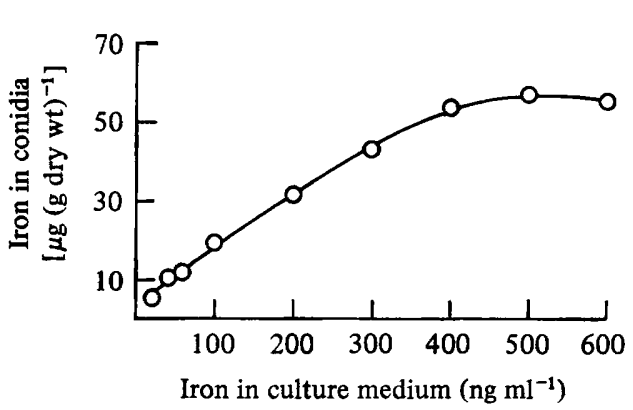

Fig. 4

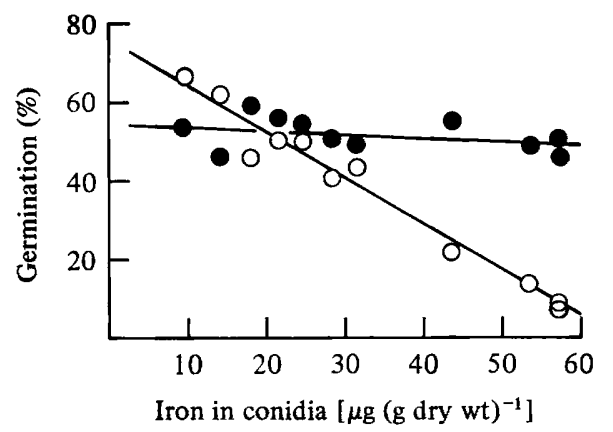

Fig. 5

Fig. 4. Influence of the iron concentration of the culture medium on the iron content of conidia.

Fig. 5. Germination as a function of the iron content of conidia, in water $(O)$ and in $10^{-3} \mathrm{M}^{-}$ anthranilic acid (O).

\section{Germination of conidia from iron-depleted media}

Mycelia and abundant conidia were produced in liquid media at all iron concentrations although growth was considerably reduced in media with less than $100 \mathrm{ng}$ iron ml-1. Conidia obtained from media containing 40 to $70 \mathrm{ng}$ iron $\mathrm{ml}^{-1}$ germinated freely in water alone (germination between 62 and $66.5 \%$ ) and the germination of such conidia was not enhanced by the addition of anthranilic acid. Increases in the concentration of iron from 70 to $500 \mathrm{ng} \mathrm{ml}^{-1}$ in the culture media on which the conidia were grown resulted in a progressive reduction in the germination of conidia in water ultimately to $9 \%$ at $500 \mathrm{ng}$ $\mathrm{ml}^{-1}$. However, in the presence of anthranilic acid germination was not affected significantly by the concentration of iron in the medium on which the conidia had been produced. A decline in percentage germination at iron concentrations below $40 \mathrm{ng} \mathrm{ml}^{-1}$ was noted and can probably be attributed to non-specific effects of severe iron deficiency on the viability of conidia.

An investigation of the iron content of the conidia used in these experiments demonstrated an approximately linear relationship between conidial iron contents and iron concentration in the culture medium up to $400 \mathrm{ng} \mathrm{ml}^{-1}$ (Fig. 4). For conidia harvested from culture media containing between 40 and $600 \mathrm{ng}^{\text {iron ml}}{ }^{-1}$ a plot of percentage germination in water against conidial iron concentration (Fig. 5) revealed a high inverse correlation $(r=0.98, P<0.001)$ between these parameters; but in the presence of anthranilic acid there was no significant relationship $(r=0.44)$ between these variables.

\section{DISCUSSION}

The synthetic chelating compounds tested showed stimulation of germination of the same order as that displayed by DHBA at comparable concentrations adding support to the hypothesis that the chelating properties of the latter are important in its biological role (Harper \& Swinburne, 1979; McCracken \& Swinburne, 1979). It is not possible to draw definitive conclusions regarding structure/activity relationships from such a limited selection of chelating agents. However, the results suggest a tendency for greater stimulatory activity to be associated with increased polarity and water solubility. Thus with 8-hydroxyquinoline germination was markedly lower than with the more polar sulphonic acid derivative, HIQS. Similarly, the introduction of hydrophobic phenyl residues into 1,10-phenanthroline led to reduced activity. This observation may reflect either increased conidial wall permeability to more polar compounds or, alternatively, may indicate that the site of action within the conidia is more accessible to compounds of this nature.

It is clear that germination begins substantially earlier in DHBA than in anthranilic acid and that the onset of germination in DHBA closely parallels that in EDTA or HIQS (Figs 1 
and 2). The delayed response with anthranilic acid is consistent with the suggestion (Harper \& Swinburne, 1979) that in order to act as a germination stimulant anthranilic acid must first undergo metabolic conversion in the conidia to DHBA. The similar response times observed with DHBA, EDTA and HIQS are strong evidence that DHBA is itself active without further metabolic conversion.

These conclusions are supported by the studies on the length of exposure to DHBA and anthranilic acid necessary to obtain maximum enhancement of conidial germination (Fig. 3). These again show that a preincubation in DHBA of $2 \mathrm{~h}$ produces a stimulation of germination comparable to a preincubation of $5 \mathrm{~h}$ with anthranilic acid.

Although the chelating agents tested have a high affinity for iron, and siderophores produced by a number of bacteria are also highly stimulatory to germination (McCracken \& Swinburne, 1979, 1980), it is not possible on this evidence to conclude that the basis of their biological action in vivo is chelation with iron rather than another metal ion. Nevertheless, convincing evidence that iron either directly or indirectly inhibits germination is provided by the following observations (Fig. 5): (i) that conidia of abnormally low iron content germinate freely in water without the normal requirement for a germination stimulant; (ii) that increasing iron content of conidia is linearly correlated with a reduction in percentage germination; (iii) that inhibition of germination is relieved at all conidial iron concentrations by anthranilic acid since, in the presence of the latter compound, percentage germination remains uniformly high at levels similar to those associated with conidia of abnormally low iron content. This inhibition is also evidently relieved non-specifically by a variety of iron-chelating agents. In vivo it is possible that the chelating agent on the banana surface is DHBA produced by conidial metabolism of anthranilic acid leached from underlying tissues. Although anthranilic acid may have some chelating ability the results of time course experiments indicating a delayed germination response to anthranilic acid compared with DHBA tend to suggest that the former compound must first be converted to DHBA before it exerts its biological action.

As to the mode of action of chelators in stimulating germination, it is clear that, since iron has an inhibitory role in germination, uptake of iron into conidia cannot be involved. This is consistent with the finding of McCracken \& Swinburne (1979) that only the desferri form of siderophores would promote germination. It must therefore be concluded that in order to stimulate germination chelators must first be incorporated into conidia where they are instrumental in the removal of iron from a binding site within the conidia and so release the germination mechanism. Whether the chelated iron is excreted from the conidia, simply transported to a site within the conidia where it is ineffective in germination inhibition, or remains in situ in the chelated form, is currently under investigation using ${ }^{59} \mathrm{Fe}$ and ${ }^{14} \mathrm{C}$-labelled chelating agents. Further work is also in progress to locate the iron involved within the conidia and elucidate the mechanism of inhibition.

H. G. was the recipient of a postgraduate fellowship awarded by the Thomas Henry Trust.

\section{REFERENCES}

HARPER, D. B. \& SwinbuRne, T. R. (1979). 2,3Dihydroxy-benzoic acid and related compounds as stimulants of germination of conidia of Colletotrichum musae (Berk. and Curt.) Arx. Physiological Plant Pathology 14, 363-370.

LANKFORD, C. E. (1973). Bacterial stimulation of iron. C.R.C. Critical Reviews in Microbiology 2, 273-331.

McCracken, A. R. \& Swinburne, T. R. (1979). Siderophores produced by saprophytic bacteria as stimulants of germination of conidia of Colletotrichum musae. Physiological Plant Pathology 15, 331-340.
McCracken, A. R. \& Swinburne, T. R. (1980). Effect of bacteria isolated from the surface of banana fruits on the germination of conidia of Colletotrichum musae (Berk. and Curt.) Arx. Transactions of the British Mycological Society 74, 18-20.

NeIlands, J. B. (1973). Microbial iron transport compounds. In Inorganic Biochemistry, pp. 167202. Edited by G. Eichkorn. Amsterdam: Elsevier.

SwinburNe, T. R. (1976). Stimulants of germination and appressoria formation by Colletotrichum musae (Berk. and Curt.) Arx in banana leachate. Phytopathologische Zeitschrift 87, 74-90. 